\title{
Prognostic value of immune related genes in lung adenocarcinoma
}

\author{
HAN WANG $^{1 *}$, MENG-SEN WANG $^{2 *}$, YING WANG $^{3 *}$, YUE-QING HUANG ${ }^{4}$ \\ JIAN-PING SHI ${ }^{5}$, ZHI-LIANG DING ${ }^{6}$ and WEN-JIE WANG ${ }^{5}$ \\ ${ }^{1}$ Department of Oncology, Jining Cancer Hospital; ${ }^{2}$ Department of Oncology, Jining First People's Hospital, Jining, \\ Shandong 272011; Departments of ${ }^{3}$ Oncology, ${ }^{4}$ General Medicine, ${ }^{5}$ Radio-Oncology and ${ }^{6}$ Neurosurgery, \\ The Affiliated Suzhou Hospital of Nanjing Medical University, Suzhou, Jiangsu 215001, P.R. China
}

Received October 17, 2019; Accepted February 7, 2020

DOI: $10.3892 / \mathrm{ol} .2020 .12122$

\begin{abstract}
Lung cancer has the highest incidence and mortality rates of all cancers in China. Immune-related genes and immune infiltrating lymphocytes are involved in tumor growth, and in the past decade, immunotherapy has become increasingly important in the treatment of lung cancer. Using the edgeR package, differentially expressed genes and immune-related genes (DEIRGs) were identified in patients with lung adenocarcinoma (LUAD). Functional enrichment analysis of DEIRGs was performed using Gene Ontology annotation and Kyoto Encyclopedia of Genes and Genomes (KEGG) pathway analyses. Survival-associated immune-related genes (IRGs) were selected using univariate Cox regression analysis and the prognostic model was assessed using multivariate Cox regression analysis. Overall, 273 DEIRGs were identified in
\end{abstract}

Correspondence to: Dr Zhi-Liang Ding, Department of Neurosurgery, The Affiliated Suzhou Hospital of Nanjing Medical University, 26 Daoqian Street, Suzhou, Jiangsu 215001, P.R. China E-mail: sz_njmy1985@163.com

Dr Wen-Jie Wang, Department of Radio-Oncology, The Affiliated Suzhou Hospital of Nanjing Medical University, 26 Daoqian Street Suzhou, Jiangsu 215001, P.R. China

E-mail: suda_wangwenjie@163.com

${ }^{*}$ Contributed equally

Abbreviations: AJCC, American Joint Committee on Cancer; CI, confidence interval; DCs, dendritic cells; DEIRGs, differentially expressed immune-related genes; GO, gene ontology; HR, hazard ratio; IRGPM, immune-related gene prognostic model; IRGs, immune-related genes; JAK-STAT, Janus kinase-signaling and transcriptional activator; KEGG, Kyoto Encyclopedia of Genes and Genomes; LUAD, lung adenocarcinoma; NSCLC, non-small cell lung cancer; OS, overall survival; PD-1, programmed death-1; PD-L1, programmed death-ligand 1; ROC, receiver operating characteristic; TCGA, The Cancer Genome Atlas; TILs, tumor-infiltrating lymphocytes

Key words: LUAD, TCGA, immune-related gene, immune infiltration, prognostic model
LUAD, and KEGG pathway analysis of IRGs showed that 'cytokine-cytokine receptor interaction' was the most significantly enriched pathway. Furthermore, six survival associated IRGs were screened to establish a prognostic model; patients in the high risk score group had less favorable survival times, and the prognostic model was negatively associated with $\mathrm{B}$ cell infiltration. The present study established a prognostic model using analysis of survival-related immune-related genes, which were associated with B cell infiltration.

\section{Introduction}

In terms of incidence and mortality rate, lung cancer ranks first among all types of cancer globally, with $<20 \%$ of patients surviving $<5$ years after diagnosis, in 2017 (1). There are two forms of lung cancer: Non-small cell lung cancer (NSCLC) and small cell lung cancer (2). NSCLC is further subdivided into lung adenocarcinoma (LUAD), squamous cell carcinoma and large cell carcinoma (3). Adenocarcinoma accounts for the largest proportion of cases, and its incidence has been increasing over the last 10 years, worldwide (4).

The primary treatments available for patients with lung cancer include surgery, chemo- and radiotherapy, molecular targeted therapy and immunotherapy. In the past few decades, researchers have improved our understanding of the role of the immune system in cancer development, and thus, immunotherapy has improved the field of tumor treatment. Of late, checkpoint inhibitors have been developed for the treatment of lung cancer $(5,6)$; blockade of immune checkpoint proteins, including programmed death-1 (PD-1)/programmed death-ligand1 (PD-L1) and cytotoxic T-lymphocyte-associated protein 4 , has shown promise in the treatment of several types of cancer, reducing tumor burden and prolonging the survival time of patients (7).

By comprehensively exploring the prognostic value of immune-related genes (IRGS), a recent study assessed individualized immune characteristics to improve the prognoses of patients with NSCLC (8). Previous studies have reported that tumor-infiltrating B cells are closely associated with a more favorable prognosis in NSCLC, cervical cancer and breast cancer (9-11). Nielsen et al (11) reported that CD20+ tumor-infiltrating lymphocytes (TILs) colocalized with activated CD8 ${ }^{+}$TILs expressed markers of antigen presentation. The group proposed 
that the association between CD20+ ${ }^{+}$TILs and patient survival may reflect a supportive role in cytolytic immune responses.

By investigating survival associated immune-related genes, the present study aimed to elucidate the underlying molecular mechanisms of immune genes in LUAD, with a view to establish therapeutic targets and provide a basis for personalized treatment.

\section{Materials and methods}

Patients. In total, 10 pairs of LUAD and adjacent normal tissues were obtained from patients with LUAD (4 men and 6 women; median age, 55 years; age range, 33-69 years), undergoing surgery at the Jining Cancer Hospital (Jining, China) from November 2018 to March 2019. None of the patients had received chemo- or radiotherapy prior to surgery. The present study was approved by the Medical Ethics Committees of Jining Cancer Hospital, and written informed consent was provided by all patients prior to surgery. A total of 497 patients were assessed from The Cancer Genome Atlas (TCGA) database (cancer.gov/tcga), including 229 men and 268 women; median age, 66 years; age range, $33-88$ years.

Data acquisition and processing. The LUAD dataset (12) containing transcriptome RNA-sequencing and clinical data of patients with LUAD was downloaded from TCGA database. A total of 497 LUAD tissues and 54 normal lung tissues were included in the present study. The list of IRGs was downloaded from the Immunology Database and Analysis Portal (ImmPort) database (13). The inclusion criteria were as follows: Patients with histologically or cytologically confirmed lung adenocarcinoma and patients with complete clinical information. The exclusion criteria were as follows: Patients with histologically or cytologically confirmed cancer other than lung adenocarcinoma and patients with OS time $<10$ days.

Identification of differentially expressed genes (DEGs), differentially expressed immune-related genes (DEIRGs) and survival-associated immune related genes (IRGs). DEGs were identified using the edgeR package (version 3.53) in Rand further analyzed. A $\log _{2}$ fold changel $>2.0$ and false discovery rate adjusted to $\mathrm{P}<0.01$ were set as the thresholds (14). In addition, volcano and heat maps of the DEGs were constructed using the gplots and heat map components of the edgeR package, respectively. DEIRGs were obtained by comparison with the immune gene lists. Survival-associated IRGs were selected using univariate Cox regression analysis, which was performed using the survival package in R.

Functional enrichment analysis. To understand the underlying biological mechanisms of the IRGs, Gene Ontology (GO) annotation and Kyoto Encyclopedia of Genes and Genomes (KEGG) pathway analyses were performed using The Database for Annotation, Visualization and Integrated Discovery (david. ncifcrf.gov/) online tool (15) and cluster profiler, an R package for functional classification and enrichment of gene clusters using the hypergeometric distribution $(16,17)$. The results of the GO and KEGG analyses were displayed using the GOplot package in $\mathrm{R}$, and analyses were based on a threshold of $\mathrm{P}<0.01$
Development of the immune-related gene prognostic model $(I R G P M)$. Overall survival time was measured from the date of diagnosis to mortality or the last clinical evaluation. Survival-associated IRGs were selected via univariate Cox regression analysis using the $\mathrm{R}$ survival package. Using multivariate Cox regression analysis via the Akaike Information Criterion (18), patients with LUAD were then divided into high-risk and low-risk groups according to the median risks core value. The risk score was calculated using the following formula:

$$
\text { Survival Risk Score }(S R S)=\sum_{i=1}^{k}\left(C_{i} \times V_{i}\right)
$$

Where $k$ represents the number of mRNAs, $C_{i}$ represents the coefficient of mRNA in multivariate Cox regression analysis and $V_{i}$ represents the mRNA expression level. Kaplan-Meier plots were used to divide patients into high and low risk score groups, according to OS time.

Relationship between IRGPM and immune cell infiltration. The Tumor Immune Estimation Resource (TIMER) online database analyzes and creates a visualization of tumor infiltrating immune cells (19). TIMER reanalyzes gene expression data, which includes 10,897 samples across 32 cancer types from TCGA, to estimate the abundance of six subtypes of tumor-infiltrating immune cells, including CD4 T cells, CD8 T cells, B cells, macrophages, dendritic cells (DCs) and neutrophils. Thus, TIMER can easily be used to determine the relationship between immune cell infiltration and other parameters. Data regarding immune infiltration levels among patients with LUAD were obtained, and the association between IRGPM and immune cell infiltration was assessed.

Reverse transcription-quantitative $(R T-q) P C R$. Total RNA was obtained from the LUAD and corresponding adjacent normal tissues of 10 patients using TRIzol $^{\circledR}$ reagent (Invitrogen; Thermo Fisher Scientific, Inc.) and then reverse transcribed into cDNA using the First Strand cDNA Synthesis kit (New England Biolabs, Inc.), according to the manufacturer's protocol. PCR amplification was performed with a SYBR Green PCR kit (ABM, Inc.), according to the manufacturer's protocol, using the Applied Biosystems 7500Real-Time PCR system (Applied Biosystems; Thermo Fisher Scientific, Inc.). The primer sequences used for qPCR are presented in Table I. The following thermocycling conditions were used for qPCR: Initial denaturation of $95^{\circ} \mathrm{C}$ for $10 \mathrm{~min} ; 40$ cycles of $95^{\circ} \mathrm{C}$ for $30 \mathrm{sec}, 60^{\circ} \mathrm{C}$ for $30 \mathrm{sec}$ and $72^{\circ} \mathrm{C}$ for $30 \mathrm{sec}$; and a final extension at $75^{\circ} \mathrm{C}$ for $7 \mathrm{~min}$. Relative mRNA expression levels were measured using the $2^{-\Delta \Delta \mathrm{Cq}}$ method (20) and normalized to the internal reference gene GAPDH. All experiments were performed in triplicate.

Statistical analysis. Survival analysis of data from patients in the prognostic model was performed using the $\mathrm{R}$ survival package. Survival curves were generated using the Kaplan-Meier method and a log-rank test was used to compare the differences between the two groups. To validate the performance of the prognostic signature, the area under the survival 
Table I. Primer sequences for reverse transcription quantitative-PCR.

\begin{tabular}{ll}
\hline Primer & \multicolumn{1}{c}{ Sequence, $5^{\prime} \rightarrow 3^{\prime}$} \\
\hline $\begin{array}{l}\text { IL11 } \\
\text { Forward }\end{array}$ & GTGGCCAAGATACAGCTGTCGC \\
Reverse & GGTAGGACAGTAGGTCCGCTC \\
$L G R 4$ & \\
Forward & TCCACCTGGAAAGTCTGA \\
Reverse & GGTTAGATTTGATTACGCTGT \\
$C R A B P 1$ & \\
Forward & ATTCTCGAGCCACCATGCCCAACTTC \\
Reverse & ACAGGATCCC TGCCTTCACTCTCGG \\
GAPDH & \\
Forward & CAACGAATTTGGCTACAGCA \\
Reverse & AGGGGTCTACATGGCAACTG \\
\hline
\end{tabular}

receiver operating characteristic (ROC) curve was calculated using the R survival ROC package (21). The expression levels of genes between different groups were evaluated using the unpaired Student's t test. $\mathrm{P}<0.05$ was considered to indicate a statistically significant difference.

\section{Results}

Identification of DEGs. Using the edgeR package, 2,672 DEGs were identified in patients with LUAD, 2,191 and 481 of which were upregulated and downregulated, respectively (Fig. 1A and B). Upon further comparison with immune gene lists from the ImmPort database, 273 DEIRGs were identified, 210 and 63 of which were up-and downregulated, respectively (Fig. 1C and D).

Construction of the prognostic model. The median gene expression was set as the cut-off value to divide all genes into two groups, high expression group and low expression group. Univariate analysis was used to identify survival-associated IRGS. The results demonstrated that high expression levels of: S100P [hazard ratio (HR), $1.218 ; 95 \%$ confidence interval (CI), 1.578-2.018; $\mathrm{P}=0.005)$, CPABP1 (HR, 1.343; 95\% CI, 1.659-2.106; P=0.005), BIRC5 (HR, 1.645; 95\% CI, 1.388-1.951; P=0.002), IGKV4-1 (HR, 1.665; 95\% CI, 1.322-2.098; P=0.009), IL11 (HR, 1.728; 95\% CI, 1.349-2.636; $\mathrm{P}<0.001)$, INHA (HR, 1.226; 95\% CI, 0.972-2.265; $\mathrm{P}=0.004)$, INSL4 (HR, 1.978; 95\% CI, 1.493-2.872; P=0.007) and LGR4 (HR, 1.678; 95\% CI, 1.433-2.172; $\mathrm{P}=0.001$ ) were associated with worse OS compared with the low expression group. Conversely, low expression levels of: ADRB2 (HR, 0.711; 95\% CI, 0.553-0.712; $\mathrm{P}=0.004)$ and VIPR1 (HR, 0.651; 95\% CI, 0.413-0.732; $\mathrm{P}<0.001)$ were associated with worse OS compared with the high expression group (Table II). Based on multivariate Cox regression analysis of survival-associated IRGs, a prognostic model was constructed which divided the patients into two groups (high risk score group and low risk score), according to OS time and the median risk score, using the following formula: (Expression levels of CRABPI*0.00326) +
Table II. Univariate Cox regression analysis of immune related genes of patients with lung adenocarcinoma.

\begin{tabular}{lcr}
\hline & \multicolumn{2}{c}{$\begin{array}{c}\text { Overall survival } \\
\text { Univariate analysis }\end{array}$} \\
\cline { 2 - 3 } Immune related & \multicolumn{1}{c}{ Hene } & P-value \\
\cline { 2 - 3 } S100P & $1.218(1.578-2.018)$ & 0.005 \\
CPABP1 & $1.343(1.659-2.106)$ & 0.005 \\
BIRC5 & $1.645(1.388-1.951)$ & 0.002 \\
IGKV4-1 & $1.665(1.322-2.098)$ & 0.009 \\
ILI1 & $1.728(1.349-2.636)$ & $<0.001$ \\
INHA & $1.226(0.972-2.265)$ & 0.004 \\
INSL4 & $1.978(1.493-2.872)$ & 0.007 \\
ADRB2 & $0.711(0.553-0.712)$ & 0.004 \\
LGR4 & $1.678(1.433-2.172)$ & 0.001 \\
VIPR1 & $0.651(0.413-0.732)$ & $<0.001$ \\
\hline
\end{tabular}

CI, confidence interval; HR, hazard ratio.

(expression levels of $\left.I G K V 4-I^{*}-0.00036\right)+($ expression levels of $I L-11 * 0.14555)+\left(\right.$ expression levels of $\left.I N H A^{*} 0.00475\right)+$ (expression levels of $\left.L G R 4^{*} 0.01757\right)+($ expression levels of $\left.V I P R I^{*}-0.17506\right)$. The results demonstrated that the expression levels of IGKV4-1 and VIPRI in high risk score group were significantly higher than low risk score group, while the expression levels of CRABPI,IL11, INHA and LGR4 in high risk score group were significantly lower than low risk score group (Fig. 2A). The risk coefficient (Fig. 2B) and mortality (Fig. 2C) were significantly higher in high risk score group compared with the low risk score group, respectively.

Gene functional enrichment analysis of differentially expressed IRGs. The biological functions of 273 IRGs were further investigated using GO and KEGG analyses. The results showed that the 'extracellular region part' was the most frequent $\mathrm{GO}$ biological process category $(\mathrm{P}<0.05$; Fig. $3 \mathrm{~A})$. The top 10 enriched GO networks and top 40 genes involved in GO networks are presented in Fig. 3A and B, respectively.

The top significantly enriched pathways were obtained using KEGG pathway analysis (Fig. 4A); these were 'cytokine-cytokine receptor interaction', 'neuroactive ligand-receptor interaction', 'JAK-STAT signaling pathway', 'IL-17 signaling pathway' and 'viral protein interaction with cytokine and cytokine receptor'. Based on the relationship between IRGs and the top 5 KEGG pathways, a visual network was constructed using Cytoscape version 3.6.1 (Fig. 4B).

Clinical outcome of patients with LUAD using the prognostic model. Kaplan-Meier plots were used to divide patients into high and low risk score groups according to OS time. The area under the ROC curve was 0.800 , suggesting that a prognostic model based on IRGs could be used to monitoring survival (Fig. 5A and B). Univariate analyses showed that high American Joint Committee on Cancer (AJCC) stage (22) (HR, 1.645; 95\% CI, 1.388-1.951; $\mathrm{P}<0.001)$, high tumor stage $(22)(\mathrm{HR}, 1.665$; 95\%; CI, 1.322-2.098; $\mathrm{P}<0.001$ ), high node stage (22) (HR, 1.928; 

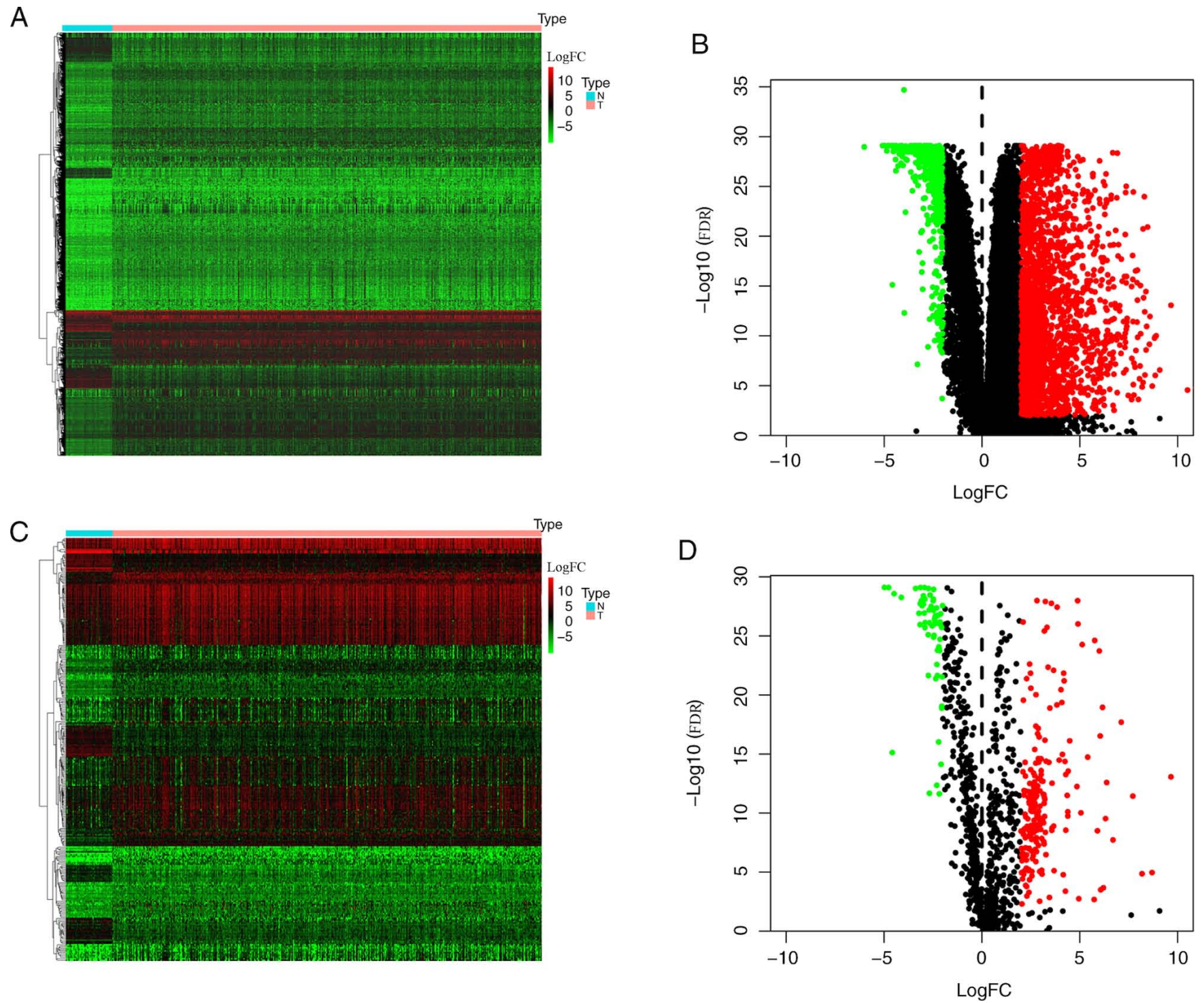

Figure 1. DEGs and DEIRGs of patients with LUAD. (A) Heatmap and (B) volcano plots of DEGs between LUAD tissues and normal tissue. (C) Heatmap and (D) volcano plots of DEIRGs between LUAD tissues and normal tissue. Red, upregulated genes; green, downregulated genes; black, nonsignificant DEGs. DEGs, differentially expressed genes; DEIRGs, differentially expressed immune-related genes; LUAD, lung adenocarcinoma; FDR, false discovery rate; $\mathrm{N}$, normal; $\mathrm{T}$, tumor.

95\% CI, 1.549-2.426; $\mathrm{P}<0.001)$ and high risk score (HR, 1.978; 95\% CI, 1.493-2.872; $\mathrm{P}<0.001)$ were significant risk factors for a poor prognosis (Table III). Using multivariate analysis, a high risks core ( $\mathrm{HR}, 2.071$; 95\% CI, 1.313-3.425; $\mathrm{P}<0.001)$ was found to be independently associated with a less favorable OS time (Table III). Collectively, these data indicate that the risk scores are significantly higher among patients with advanced $\mathrm{T}$ (Fig. 5C) and high AJCC stages (Fig. 5D).

Correlation analysis of the prognostic model and immune cell infiltration. Among the six immune cell types investigated (B cells, CD4 T cells, CD8 T cells, DCs, macrophages and neutrophils), the risk factors identified in the prognostic model were negatively correlated with B cell infiltration $(r=-0.158$; $\mathrm{P}=0.001$; Fig. 6A); however, risk score was not associated with $\mathrm{CD}^{+} \mathrm{T}$ cells $\left(\mathrm{r}=-0.078 ; \mathrm{P}=0.112\right.$; Fig. 6B), $\mathrm{CD} 8^{+} \mathrm{T}$ cells $(r=-0.015 ; \mathrm{P}=0.756$; Fig. $6 \mathrm{C})$, dendritic cells $(\mathrm{r}=-0.080$; $\mathrm{P}=0.102$; Fig. 6D), macrophages $(\mathrm{r}=-0.068 ; \mathrm{P}=0.164$; Fig. $6 \mathrm{E})$ or neutrophils $(\mathrm{r}=0.018 ; \mathrm{P}=0.715$; Fig. $6 \mathrm{~F})$.
Analysis and validation of gene expression. To further validate the expression of relevant key genes in the prognostic model, three mRNAs (IL11, CARBP1 and LGR4) were randomly selected and their expression levels were evaluated in 10 pairs of LUAD and adjacent normal tissues. The expression levels of IL11, CRABP1 and LGR4 were higher in tumor tissues compared with adjacent normal tissues (Fig. 7A-C), which was consistent with the findings observed in TCGA database.

\section{Discussion}

The incidence and mortality rates of lung cancer in China are still increasing (23). A previous study indicated that IRGs are promising prognostic indicators of early stage lung cancer (8). A particular study screened for 40 genes and classified patients into high-risk and low-risk groups according to the immune signature; Patients with early non-squamous lung cancer demonstrated independent prognostic factors (8). As is well known, the treatment of advanced stage unresectable or metastatic 
Table III. Univariate and Multivariate Cox regression analysis of prognostic model (risk score) and clinical features of patients with lung adenocarcinoma.

Overall survival

\begin{tabular}{cccc}
\hline \multicolumn{2}{c}{ Univariate analysis } & \multicolumn{2}{c}{ Multivariate analysis } \\
\hline HR $(95 \% \mathrm{CI})$ & P-value & HR $(95 \% \mathrm{CI})$ & P-value \\
$0.998(0.978-1.018)$ & 0.842 & $1.002(0.981-1.023)$ & 0.863 \\
$0.843(0.659-1.406)$ & 0.843 & $0.864(0.581-1.286)$ & 0.472 \\
$1.645(1.388-1.951)$ & $<0.001$ & $1.547(0.909-2.640)$ & 0.108 \\
$1.665(1.322-2.098)$ & $<0.001$ & $1.280(0.985-1.662)$ & 0.065 \\
$1.928(1.549-2.426)$ & $<0.001$ & $1.281(0.795-2.062)$ & 0.309 \\
$1.226(0.972-2.265)$ & 0.096 & $1.408(0.541-1.966)$ & 0.277 \\
$1.978(1.493-2.872)$ & $<0.001$ & $2.071(1.313-3.425)$ & $<0.001$ \\
\hline
\end{tabular}

AJCC, American Joint Committee on Cancer; CI, confidence interval; HR, hazard ratio; M, metastasis; N, Node; T, Tumor.
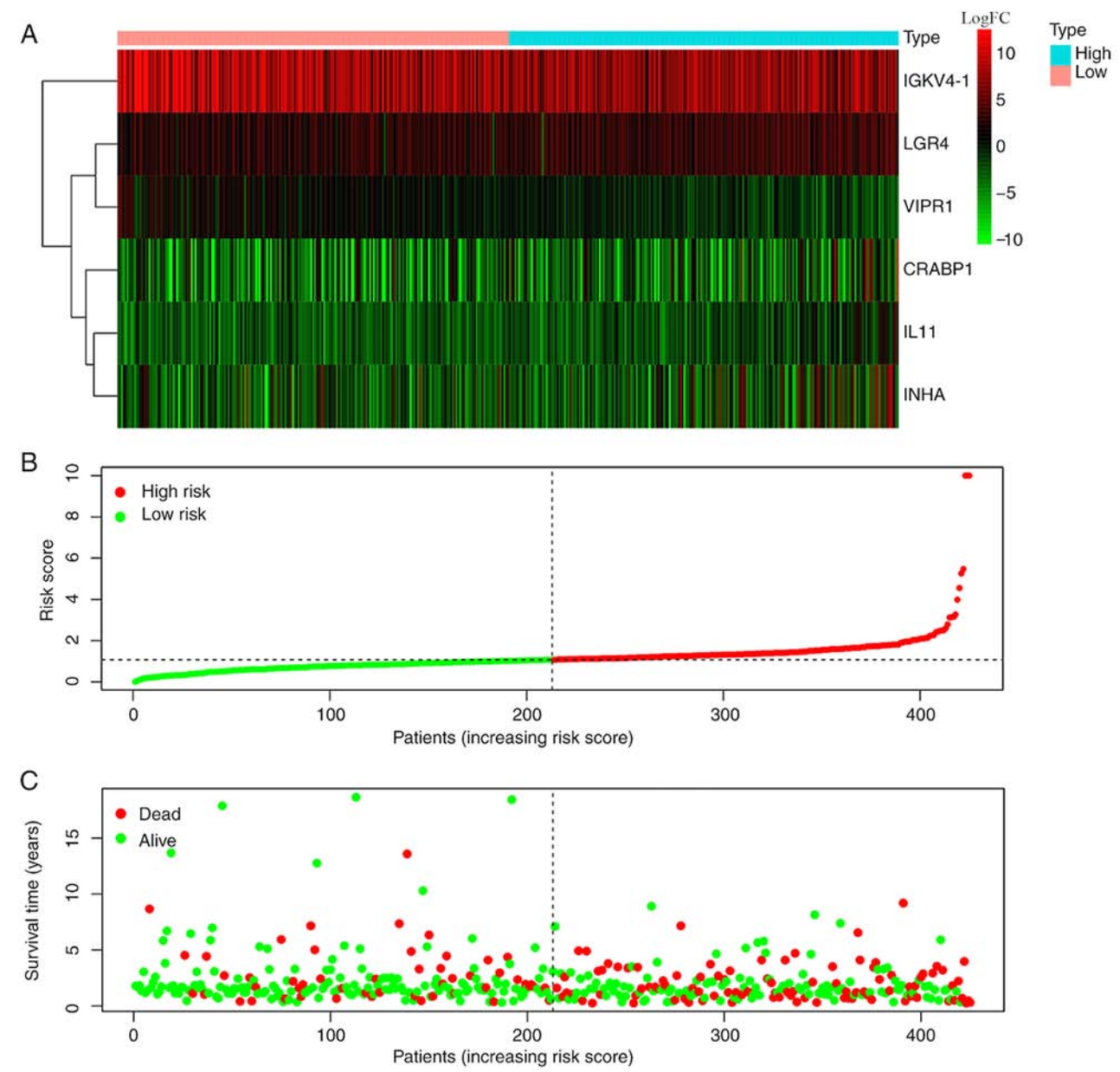

Figure 2. Construction of a prognostic model using survival-associated, immune-related genes from patients with lung adenocarcinoma. (A) Heatmap of survival-associated immune-related genes in the prognostic model. Distribution of different (B) risk scores and (C) survival status for each patient. 
A GO:0044421 extracellular region part
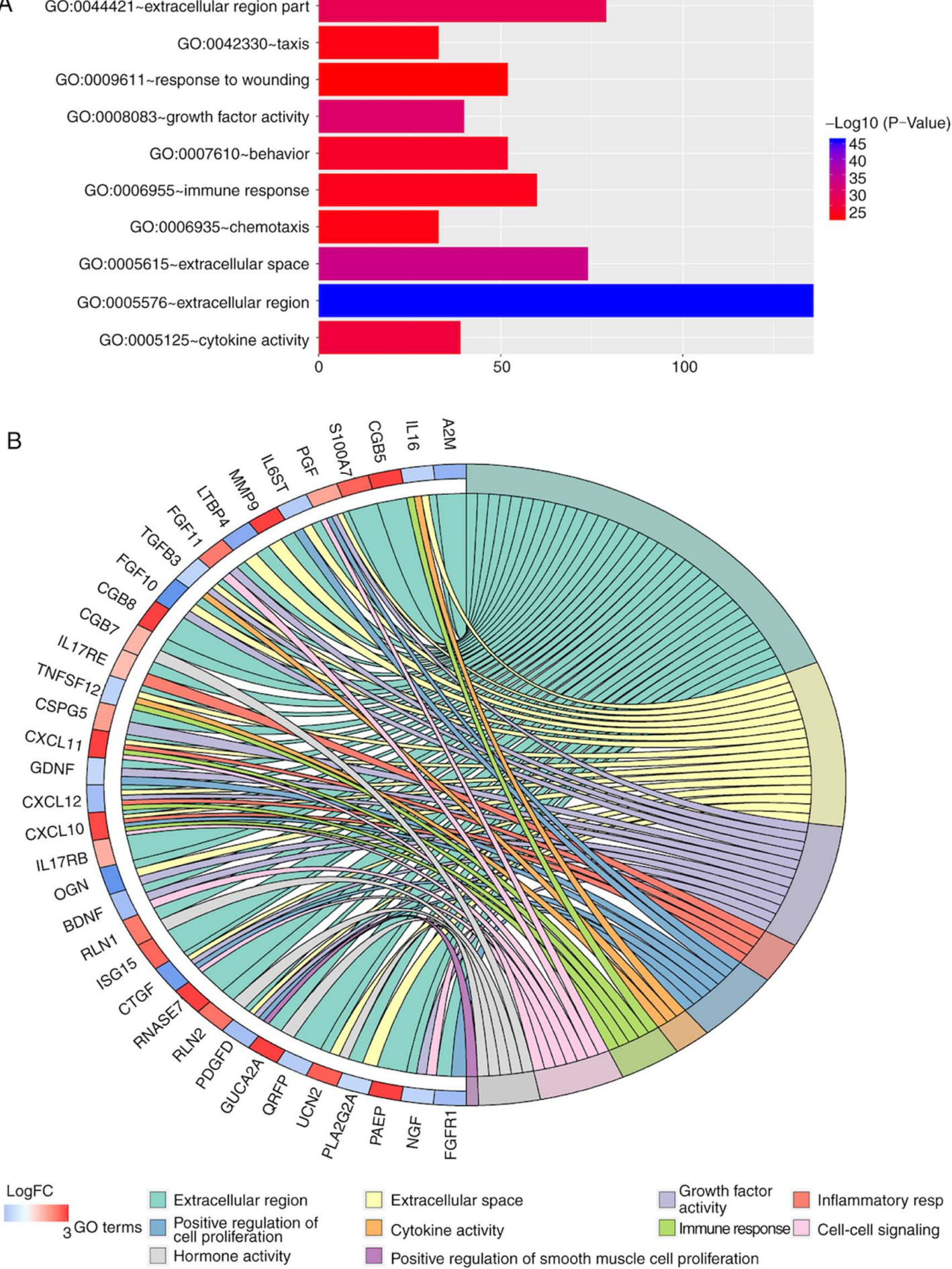

Figure 3. Gene functional enrichment analysis of differentially expressed immune-related genes of patients with lung adenocarcinoma. (A) GO biological process categories. (B) Top 40 enriched genes involved in the GO networks. GO, Gene Ontology; FC, fold-change.

lung cancer in China is difficult (24). Immunotherapy, such as immune checkpoint inhibitors, is primarily used for patients with metastatic lung cancer $(5,6)$; therefore, the present study aimed to predict the prognoses of patients with LUAD using IRGs.

A total of six IRGs associated with prognosis were identified in patients with LUAD using TCGA database, and a prognostic model was established based on these genes (CRABPI,IGKV4-1, ILII, INHA, LGR4 and VIPRI). In this model, the overall survival duration of patients with high-risk disease was significantly shorter compared with patients with low-risk disease.

$C R A B P 1$ is a member of the fatty acid binding family of proteins, which binds to retinoic acid with high affinity (25). There are few studies on $C R A B P 1$ and lung cancer and the underlying molecular mechanisms of $C R A B P 1$ function in lung cancer remain unclear. Favorskaya et al demonstrated thatCRABP1 significantly alters the expression levels of $C R A B P$ in 


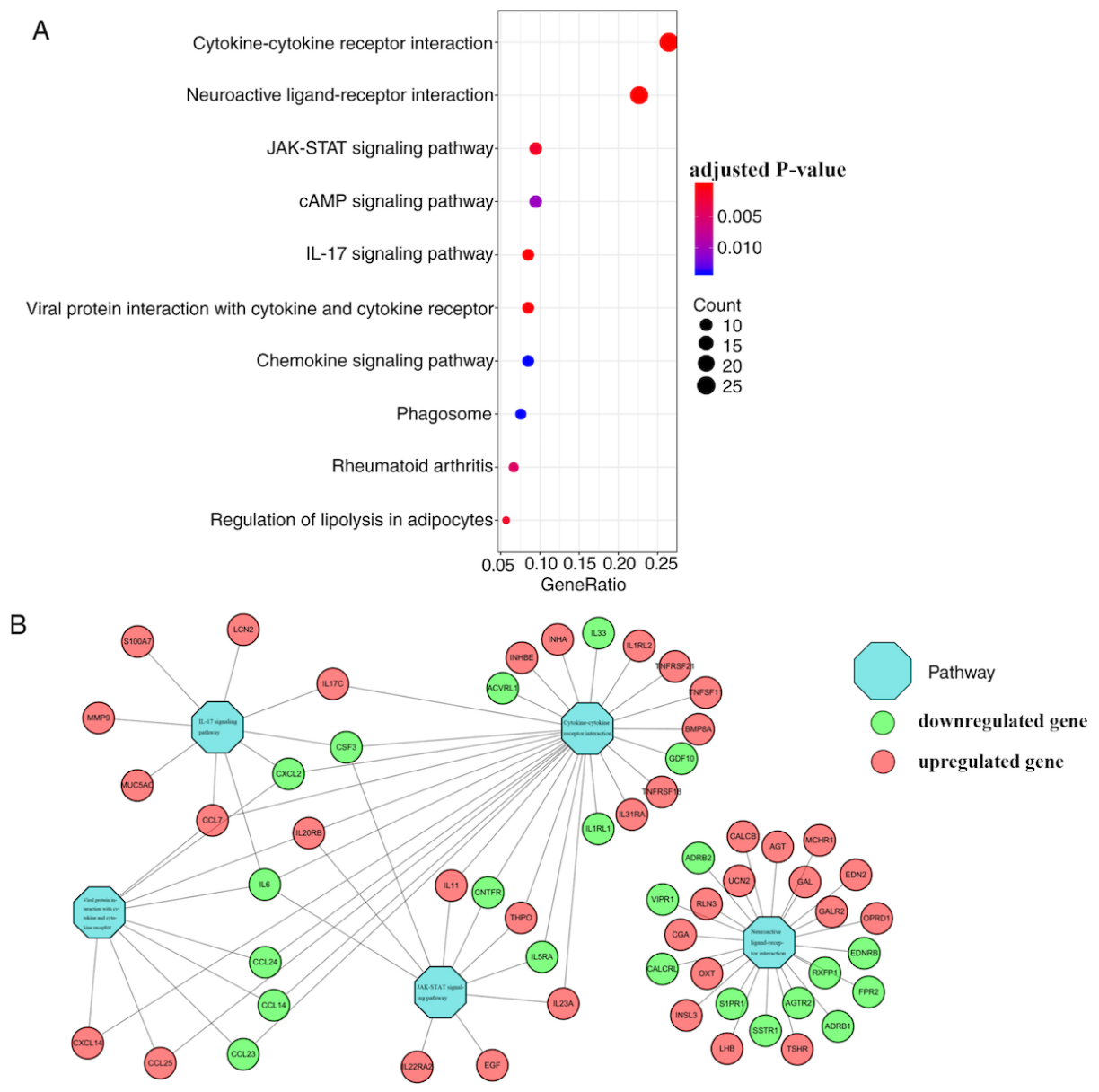

Figure 4. The KEGG pathway of differentially expressed immune-related genes of patients with lung adenocarcinoma. (A) Top 10 significantly enriched KEGG pathways. (B) Visual network of the relationships between immune-related genes and the top5 KEGG pathways. KEGG, Kyoto Encyclopedia of Genes and Genomes.

A

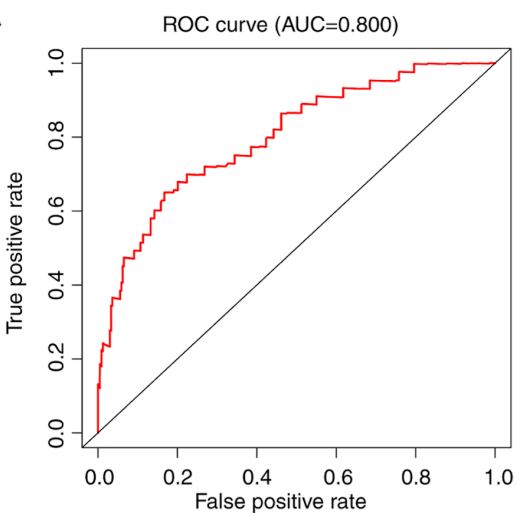

C

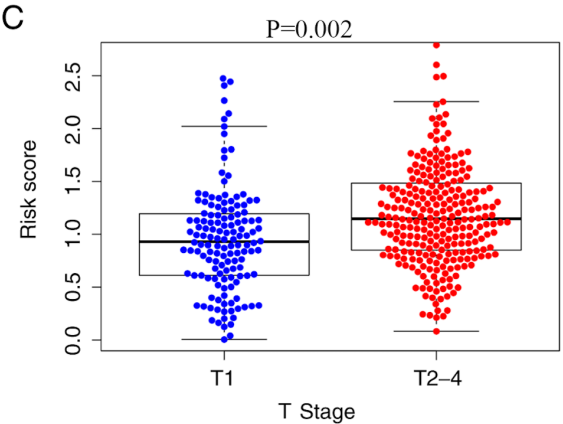
Risk + High risk + Low risk
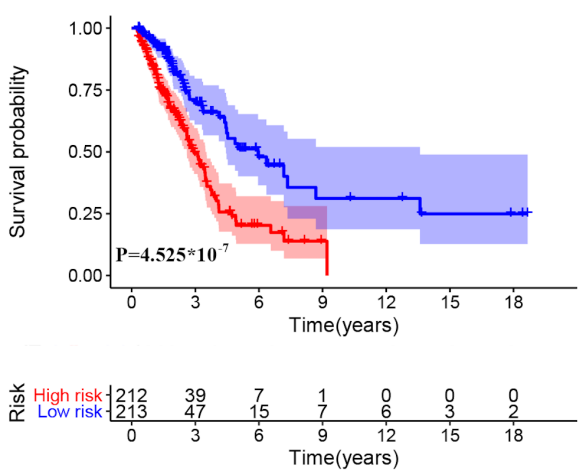

D

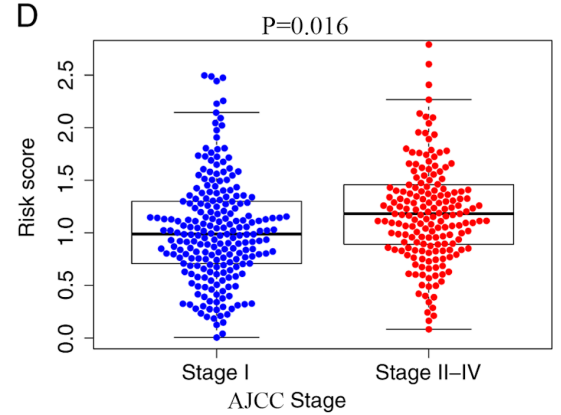

Figure 5. Survival analysis of prognostic model of patients with lung adenocarcinoma. Blue represents the low risk group and red represents the high-risk group. (A) ROC curve analysis of prognostic model. (B) Kaplan-Meier analysis between high risk and low risk score groups. (C) Relationships between risk score and T stage. (D) Relationships between risk score and American Joint Committee on Cancer stage. ROC, receiver operating characteristic; AUC, area under the curve; T, tumor. 
A

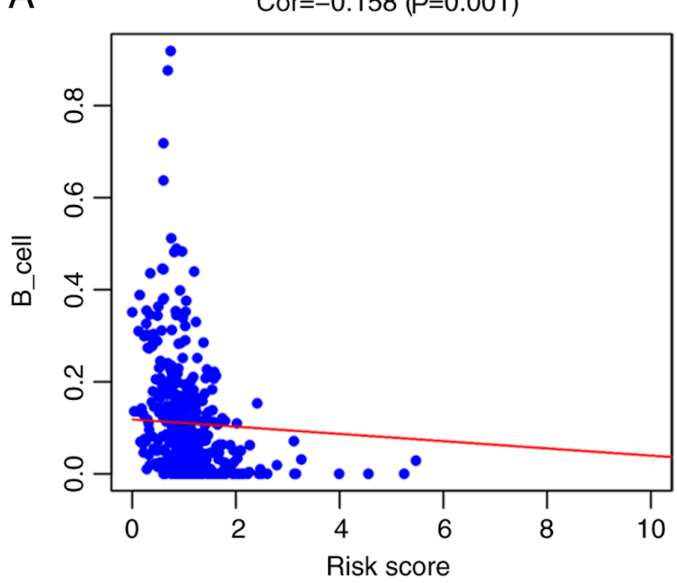

C

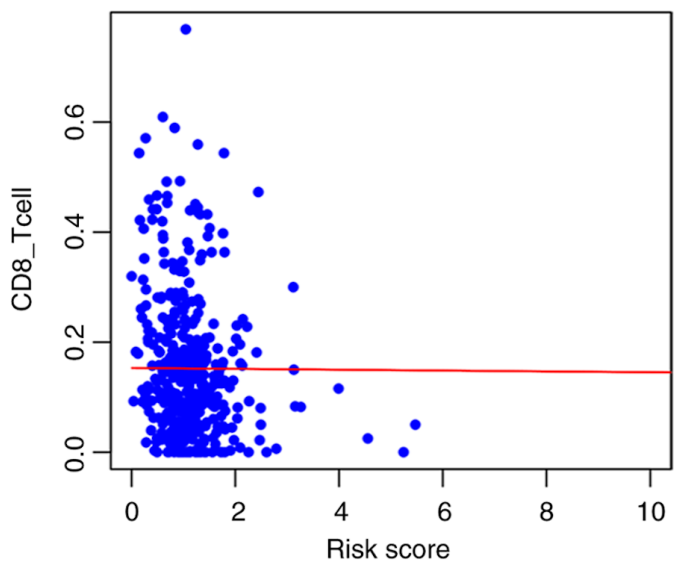

E

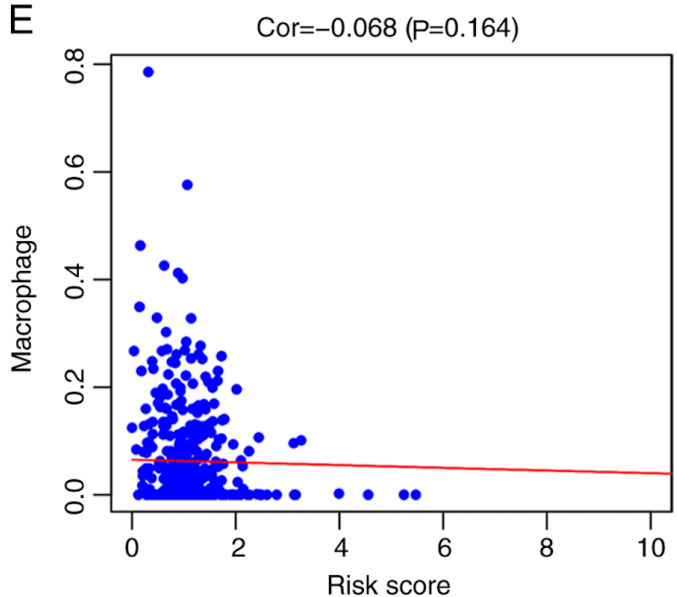

B

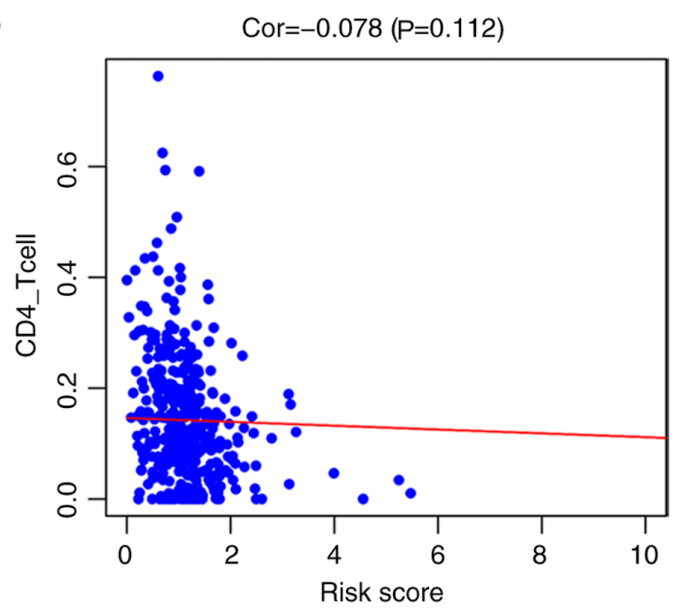

D

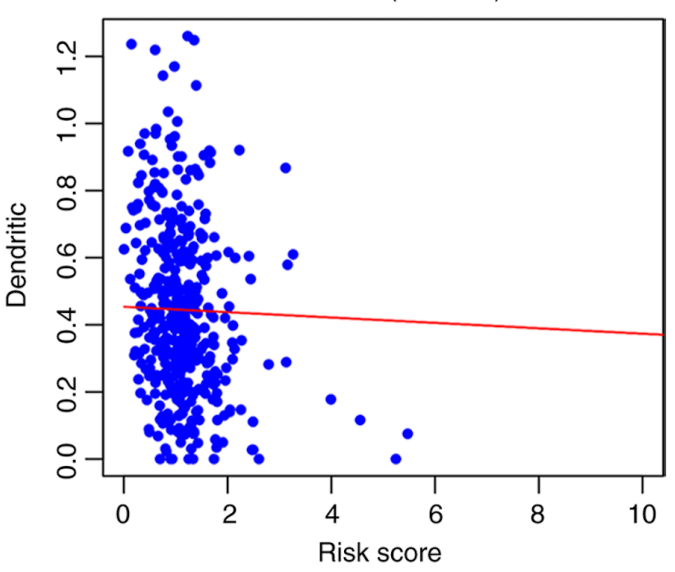

$\mathrm{F}$

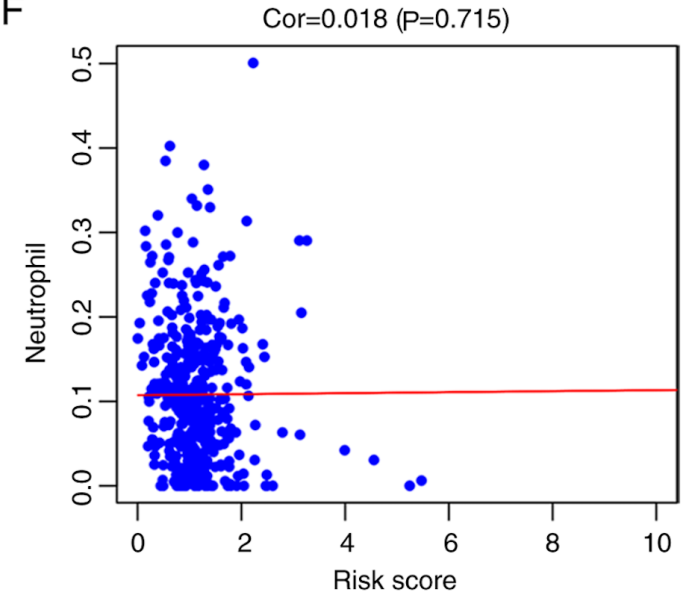

Figure 6. Relationship between prognostic model (risk score) and infiltration of several immune cells. Infiltration of (A) B cells, (B) CD4 T cells, (C) CD8 T cells, (D) dendritic cells, (E) macrophages and (F) neutrophils. Cor, correlation.

A

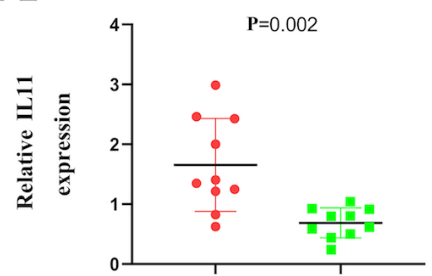

$\mathrm{B}$

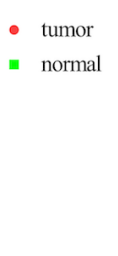

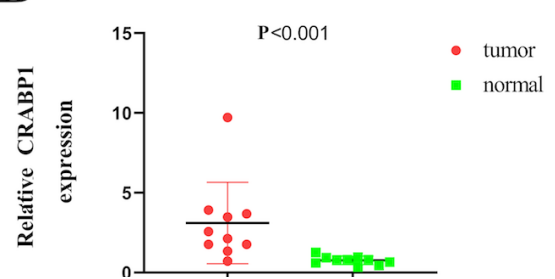

$\mathrm{C}$

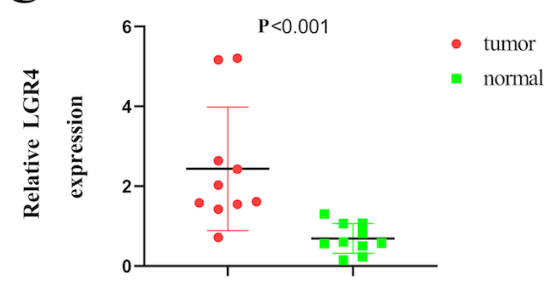

Figure 7. mRNA expression levels of $I L 11, C R A B P 1$ and $L G R 4$ between tumor tissues and adjacent tissues of patients with lung adenocarcinoma. Expression levels of (A) IL11, (B) CRABP1 and (C) LGR4. 
NSCLC samples (26). IGKV4-1 is inherently autoreactive and has been implicated in B-cell mediated autoimmune diseases and dysregulated B-cell tolerance (27-29). However, the function of IGKV4-1 in lung cancer remains unknown. VIPR1 is a $\mathrm{G}$ protein-coupled receptor that is widely distributed in the normal tissues of humans, and that serves a role in physiological functions (30). Downregulation and deletion of VIPRI have been detected in patients with LUAD (31). Gong et al (32) demonstrated that $L G R 4$ was expressed in LUAD tissues but not in normal lung tissue. The group reported that $L G R 4$ and $I Q G A P 1$ served a role in the regulation of tumor growth and metastasis in lung cancer cells. $I L-11$ is a member of the $I L-6$ group and binds to its corresponding receptors. $I L-11$ is an important inflammatory mediator that can affect the activity of a variety of immune cells (33-35). Increased $I L-11$ expression levels have been associated with various types of cancer, including LUAD (36-38).

To further explain some of these potential mechanisms, gene functional enrichment analysis was performed. It was demonstrated that IRGs were primarily enriched in 'cytokine-cytokine receptor interaction', 'neuroactive ligand-receptor interaction' and the 'JAK-STAT signaling pathway'. Among the above prognosis-related immune genes, $I L-11$ was associated with "cytokine-cytokine receptor interaction' and the 'JAK-STAT signaling pathway'.

Cytokines are secreted glycoproteins that function as intercellular mediators, promoting cellular proliferation, differentiation and apoptosis (39). On the other hand, cytokines secreted by tumors can promote the recruitment of immunosuppressive cells, resulting in tumor metastasis (40). Previous studies have identified a variety of cytokines that can regulate hematopoiesis, induce inflammatory responses and control immune responses through the Janus kinase (JAK) signaling pathway $(41,42)$. The JAK family contains four members: JAK1, JAK2, JAK3 and TYK2 (43). JAK kinases are a potential target for the treatment of tumors due to the oncogenic effects and the promotion of tumor inflammatory responses via JAK signaling (41). When cytokines bind to their cognate receptors, JAK is activated and phosphorylates downstream signaling and transcriptional activator (STAT), ultimately leading to tumor invasion, angiogenesis, apoptosis and metastasis (41). $I L-11$ activates downstream JAK/STAT signaling proteins via a gp130 homodimer (42). The suppressor of cytokine signaling proteins regulate JAK/STAT signaling pathways by serving as feedback inhibitors of activated JAK (44). Currently, few studies have investigated $I L-11$ and JAK signaling pathways in tumors, and the underlying molecular mechanisms of action remain unknown. In the present study, KEGG analysis revealed that IRGs are mainly enriched in these two signaling pathways. Further network construction revealed that $I L-11$ is closely associated with these two pathways. The explanation of this relationship between the two pathways in the present study may provide a basis for determining the prognosis of lung cancer.

In the present study, the immune gene-related prognostic index was not only associated with the prognosis of LUAD but was also negatively correlated with immune B cell infiltration. Tumor-infiltrating B lymphocytes have seemingly conflicting effects in tumors. On the one hand, B cells function in the inhibition of tumor cell proliferation via antigen restricted tumoricidal responses; on the other hand, $\mathrm{B}$ cells also act by suppressing the immune system, thus promoting tumor growth, proliferation and metastasis (45). Tumor-infiltrating B lymphocyte-derived lymphotoxin has been reported to promote the progression of androgen-independent prostate cancer by activating the Nuclear Factor $\mathrm{k}-\mathrm{B}$ and STAT3 signaling pathways (46). Previous studies have shown that tumor-infiltrating $\mathrm{CD} 20^{+} \mathrm{B}$ cells reside in close proximity to $\mathrm{CD}^{+} \mathrm{T}$ cells, and in patients with ovarian cancer, infiltration of $\mathrm{CD} 20^{+} \mathrm{B}$ and $\mathrm{CD} 8^{+} \mathrm{T}$ cells prolongs $\mathrm{DSS}$ (disease-specific survival) compared with $\mathrm{CD}^{+} \mathrm{T}$ cell infiltration alone (11). Pinto et al (47) demonstrated that patients with LUAD, with mutated $K-R A S$ had associated B cell infiltration. B cells also exert a number of anti-tumor effects. First, they can stimulate other immune cells to produce cytokines, particularly those that enhance the activity of CTL (cytotoxic T-lymphocytes) (48). Secondly, B cells secrete granzyme B to directly kill tumor cells (48). Furthermore, B cells can suppress pancreatic cancer via antibody-dependent mechanisms $(48,49)$. However, the functions of a number of prognosis-related genes in lung cancer remain unclear. For example, the IGKV4-I gene encodes a B cell receptor (50). Previous studies have not described the relationship between IGKV4-1 and lung cancer. It is unclear whether the IGKV4-1 gene serves a role in B cell infiltration in lung cancer, and further research is required to investigate the possible underlying molecular mechanisms. Due to the negative correlation between the prognostic model and B cell infiltration in the present study, some patients may have had low risk scores due to the anti-tumor effects exerted by infiltrating B lymphocytes.

The present study was not without limitations. The primary limitation was the small sample size, which will be increased in future studies. Although the conclusions of the present study were drawn based on evidence from TCGA database, only gene expression was verified. Thus, it remains critical to further verify the applicability of survival models.

In summary, the present study identified prognosis-related immune genes using TCGA database and established a prognostic model for patients with LUAD. Using multivariate analysis with other clinicopathological features, such as age, gender and TNM stage, risk score was revealed to be an independent prognostic factor, hence, the present model can predict the prognoses of patients with LUAD. In addition, the prognostic model was associated with B cell infiltration and the present study may provide novel evidence for the prognosis and immunotherapy of patients with LUAD in the future.

\section{Acknowledgements}

The authors would like to thank Dr Wei Shan (Department of Gastrointestinal Surgery, Renmin Hospital of Wuhan University) for his assistance regarding statistics.

\section{Funding}

The present study was supported by The Science and Education for Health Foundation of Suzhou for Youth (grant nos. kjxw2018030 and kjxw2018032), The Science and Technology Project Foundation of Suzhou (grant no. SS201651), The Education Research Project Foundation of Nanjing Medical University (grant no. FZS-ZD-201701), The Jiangsu 
Province Medical Key Discipline (grant no. ZDXKC2016007) and Suzhou Oncology Clinical Center (grant no. Szzx201506).

\section{Availability of data and materials}

The datasets generated and analyzed during the current study are available in The Cancer Genome Atlas repository (https://portal.gdc.cancer.gov/).

\section{Authors' contributions}

ZLD and WJW conceived and designed the study. YQH and JPS performed the statistical analysis. HW, MSW and YW were involved in the writing of the manuscript and in the interpretation of the results. All authors read and approved the final manuscript.

\section{Ethics approval and consent to participate}

The present study was approved by The Medical Ethics Committees of Jining Cancer Hospital (approval no. 20190067). Written informed consent was provided by all patients prior to the study start.

\section{Patient consent for publication}

Not applicable.

\section{Competing interests}

The authors declare that they have no competing interests.

\section{References}

1. Siegel RL, Miller KD and Jemal A: Cancer statistics, 2017. CA Cancer J Clin 67: 7-30, 2017.

2. Sher T, Dy GK and Adjei AA: Small cell lung cancer. Mayo Clin Proc 83: 355-367, 2008

3. Duffy MJ and O'Byrne K: Tissue and blood biomarkers in lung cancer: A review. Adv Clin Chem 86: 1-21, 2018.

4. Meza R, Meernik C, Jeon J and Cote ML: Lung cancer incidence trends by gender, race and histology in the United States, 1973-2010. PLoS One 10: e0121323, 2015.

5. Garon EB, Rizvi NA, Hui R, Leighl N, Balmanoukian AS, Eder JP, Patnaik A, Aggarwal C, Gubens M, Horn L, et al: Pembrolizumab for the treatment of non-small-cell lung cancer. N Engl J Med 372: 2018-2028, 2015.

6. Hellmann MD, Rizvi NA, Goldman JW, Gettinger SN, Borghaei H, Brahmer JR, Ready NE, Gerber DE, Chow LQ, Juergens RA, et al: Nivolumab plus ipilimumab as first-line treatment for advanced non-small-cell lung cancer (CheckMate 012): Results of an open-label, phase 1, multicohort study. Lancet Oncol 18: 31-41, 2017

7. Postow MA, Callahan MK and Wolchok JD: Immune checkpoint blockade in cancer therapy. J Clin Oncol 33: 1974-1982, 2015.

8. Li B, Cui Y, Diehn M and Li R: Development and validation of an individualized immune prognostic signature in early-stage nonsquamous non-small cell lung cancer. JAMA Oncol 3: 1529-1537, 2017.

9. Al-Shibli KI, Donnem T, Al-Saad S, Persson M, Bremnes RM and Busund LT: Prognostic effect of epithelial and stromal lymphocyte infiltration in non-small cell lung cancer. Clin Cancer Res 14: 5220-5227, 2008.

10. Nedergaard BS, Ladekarl M, Nyengaard JR and Nielsen K: A comparative study of the cellular immune response in patients with stage IB cervical squamous cell carcinoma. Low numbers of several immune cell subtypes are strongly associated with relapse of disease within 5 years. Gynecol Oncol 108: 106-111, 2008 .
11. NielsenJS,SahotaRA,MilneK,KostSE,NesslingerNJ,WatsonPH and Nelson BH: CD20+ tumor -infiltrating lymphocytes have an atypicalCD27-memory phenotypeand together withCD8+Tcells promote favorable prognosis in ovarian cancer. Clin Cancer Res 18: 3281-3292, 2012.

12. Liu J, Lichtenberg T, Hoadley KA, Poisson LM, Lazar AJ, Cherniack AD, Kovatich AJ, Benz CC, Levine DA, Lee AV, et al: An integrated TCGA pan-cancer clinical data resource to drive high-quality survival outcome analytics. Cell 173: 400-416.e11, 2018.

13. Bhattacharya S, Andorf S, Gomes L, Dunn P, Schaefer H, Pontius J, Berger P, Desborough V, Smith T, Campbell J, et al: ImmPort: Disseminating data to the public for the future of immunology. Immunol Res 58: 234-239, 2014.

14. Robinson MD, McCarthy DJ and Smyth GK: EdgeR: A bioconductor package for differential expression analysis of digital gene expression data. Bioinformatics 26: 139-140, 2010.

15. Dennis G Jr, Sherman BT, Hosack DA, Yang J, Gao W, Lane HC and Lempicki RA: DAVID: Database for annotation, visualization, and integrated discovery. Genome Biol 4: P3, 2003.

16. Yu G, Wang LG, Han Y and He QY: ClusterProfiler: An R package for comparing biological themes among gene clusters. OMICS 16: 284-287, 2012.

17. Huang da W, Sherman BT and Lempicki RA: Bioinformatics enrichment tools: Paths toward the comprehensive functional analysis of large gene lists. Nucleic Acids Res 37: 1-13, 2009.

18. Wagenmakers EJ and Farrell S: AIC model selection using Akaike weights. Psychon Bull Rev 11: 192-196, 2004.

19. Li T, Fan J, Wang B, Traugh N, Chen Q, Liu JS, Li B and Liu XS: TIMER: A web server for comprehensive analysis of tumor-infiltrating immune cells. Cancer Res 77: e108-e110, 2017.

20. Livak KJ and Schmittgen TD: Analysis of relative gene expression data using real-time quantitative PCR and the 2(-Delta Delta C(T)) method. Methods 25: 402-408, 2001.

21. Heagerty PJ, Lumley T and Pepe MS: Time-dependent ROC curves for censored survival data and a diagnostic marker. Biometrics 56: 337-344, 2000.

22. Detterbeck FC, Boffa DJ, Kim AW and Tanoue LT: The eighth edition lung cancer stage classification. Chest 151: 193-203, 2017.

23. Chen W, Zheng R, Baade PD, Zhang S, Zeng H, Bray F, Jemal A, $\mathrm{Yu}$ XQ and He J: Cancer statistics in China, 2015. CA Cancer J Clin 66: 115-132, 2016.

24. Chinese Association for Clinical Oncologists: Clinical practice guideline for stage IV primary lung cancer in China(2020 version). Zhonghua Zhong Liu Za Zhi 42: 1-16, 2020 (In Chinese).

25. Dong D, Ruuska SE, Levinthal DJ and Noy N: Distinct roles for cellular retinoic acid-binding proteins I and II in regulating signaling by retinoic acid. J Biol Chem 274: 23695-23698, 1999.

26. Favorskaya I, Kainov Y, Chemeris G, Komelkov A, Zborovskaya I and Tchevkina E: Expression and clinical significance of CRABP1 and CRABP2 in non-small cell lung cancer. Tumour Biol 35: 10295-10300, 2014.

27. Yurasov S, Wardemann H, Hammersen J, Tsuiji M, Meffre E, Pascual V and Nussenzweig MC: Defective B cell tolerance checkpoints in systemic lupus erythematosus. J Exp Med 201: 703-711, 2005.

28. Pugh-Bernard AE, Silverman GJ, Cappione AJ, Villano ME, Ryan DH, Insel RA and Sanz I: Regulation of inherently autoreactive VH4-34 B cells in the maintenance of human B cell tolerance. J Clin Invest 108: 1061-1070, 2001.

29. Cappione A III, Anolik JH, Pugh-Bernard A, Barnard J, Dutcher P, Silverman G and Sanz I: Germinal center exclusion of autoreactive B cells is defective in human systemic lupus erythematosus. J Clin Invest 115: 3205-3216, 2005.

30. Reubi JC, Laderach U, Waser B, Gebbers JO, Robberecht P and Laissue JA: Vasoactive intestinal peptide/pituitary adenylate cyclase-activating peptide receptor subtypes in human tumors and their tissues of origin. Cancer Res 60: 3105-3112, 2000.

31. Mlakar V, Strazisar M, Sok M and Glavac D: Oligonucleotide DNA microarray profiling of lung adenocarcinoma revealed significant downregulation and deletions of vasoactive intestinal peptide receptor 1. Cancer Invest 28: 487-494, 2010.

32. Gong X, Yi J, Carmon KS, Crumbley CA, Xiong W, Thomas A, Fan X, Guo S, An Z, Chang JT and Liu QJ: Aberrant RSPO3-LGR4 signaling in Keap1-deficient lung adenocarcinomas promotes tumor aggressiveness. Oncogene 34: 4692-4701, 2015. 
33. Zhang J, Zhang Y, Dutta DJ, Argaw AT, Bonnamain V, Seto J, Braun DA, Zameer A, Hayot F, Lòpez CB, et al: Proapoptotic and antiapoptotic actions of Stat1 versus Stat3 underlie neuroprotective and immunoregulatory functions of IL-11. J Immunol 187: 1129-1141, 2011.

34. Trepicchio WL, Wang L, Bozza M and Dorner AJ: IL-11 regulates macrophage effector function through the inhibition of nuclear factor-kappaB. J Immunol 159: 5661-5670, 1997.

35. Curti A, Ratta M, Corinti S, Girolomoni G, Ricci F, Tazzari P, Siena M, Grande A, Fogli M, Tura S and Lemoli RM Interleukin-11 induces Th2 polarization of human CD4(+) T cells. Blood 97: 2758-2763, 2001.

36. Pastor MD, Nogal A, Molina-Pinelo S, Quintanal-Villalonga Á, Meléndez R, Ferrer I, Romero-Romero B, De Miguel MJ, López-Campos JL, Corral J, et al: IL-11 and CCL-1: Novel protein diagnostic biomarkers of lung adenocarcinoma in bronchoalveolar lavage fluid (BALF). J Thorac Oncol 11: 2183-2192, 2016.

37. Yamazumi K, Nakayama T, Kusaba T, Wen CY, Yoshizaki A, Yakata Y, Nagayasu T and Sekine I: Expression of interleukin-11 and interleukin-11 receptor alpha in human colorectal adenocarcinoma; immunohistochemical analyses and correlation with clinicopathological factors. World J Gastroenterol 12: 317-321, 2006.

38. Hanavadi S, Martin TA, Watkins G, Mansel RE and Jiang WG: Expression of interleukin 11 and its receptor and their prognostic value in human breast cancer. Ann Surg Oncol 13: 802-808, 2006.

39. Morris R, Kershaw NJ and Babon JJ: The molecular details of cytokine signaling via the JAK/STAT pathway. Protein Sci 27: 1984-2009, 2018.

40. Hanahan D and Coussens LM: Accessories to the crime: Functions of cells recruited to the tumor microenvironment. Cancer Cell 21: 309-322, 2012.

41. O'Shea JJ, Schwartz DM, Villarino AV, Gadina M, McInnes IB and Laurence A: The JAK-STAT pathway: Impact on human disease and therapeutic intervention. Annu Rev Med 66: 311-328, 2015.

42. Buzzelli JN, O'Connor L, Scurr M, Chung Nien Chin S, Catubig A $\mathrm{Ng} \mathrm{GZ,} \mathrm{Oshima} \mathrm{M,} \mathrm{Oshima} \mathrm{H,} \mathrm{Giraud} \mathrm{AS,} \mathrm{Sutton} \mathrm{P,} \mathrm{et} \mathrm{al:}$ Overexpression of IL-11 promotes premalignant gastric epithelial hyperplasia in isolation from germline gp130-JAK-STAT driver mutations. Am J Physiol Gastrointest Liver Physiol 316 : G251-G262, 2019.
43. Stark GR and Darnell JE Jr: The JAK-STAT pathway at twenty. Immunity 36: 503-514, 2012.

44. Inagaki-Ohara K, Kondo T, Ito M and Yoshimura A: SOCS, inflammation, and cancer. JAKSTAT 2: e24053, 2013.

45. Guo S, Contratto M, Miller G, Leichman L and $\mathrm{Wu} \mathrm{J}$ : Immunotherapy in pancreatic cancer: Unleash its potential through novel combinations. World J Clin Oncol 8: 230-240, 2017.

46. Ammirante M, Luo JL, Grivennikov S, Nedospasov S and Karin M: B-cell-derived lymphotoxin promotes castration-resistant prostate cancer. Nature 464: 302-305, 2010.

47. Pinto R, Petriella D, Lacalamita R, Montrone M, Catino A, Pizzutilo P, Botticella MA, Zito FA, Del Bene G, Zonno A, et al: KRAS-driven lung adenocarcinoma and $B$ cell infiltration: Novel insights for immunotherapy. Cancers (Basel) 11: pii: E1145, 2019.

48. Tsou P, Katayama H, Ostrin EJ and Hanash SM: The emerging role of B cells in tumor immunity. Cancer Res 76: 5597-5601, 2016.

49. Lee KE, Spata M, Bayne LJ, Buza EL, Durham AC, Allman D, Vonderheide RH and Simon MC: Hif 1a deletion reveals pro-neoplastic function of B cells in pancreatic neoplasia. Cancer Discov 6: 256-269, 2016.

50. Asare A, Kanaparthi S, Lim N, Phippard D, Vincenti F, Friedewald J, Pavlakis M, Poggio E, Heeger P, Mannon R, et al: $\mathrm{B}$ cell receptor genes associated with tolerance identify a cohort of immunosuppressed patients with improved renal allograft graft function. Am J Transplant 17: 2627-2639, 2017.

This work is licensed under a Creative Commons Attribution-NonCommercial-NoDerivatives 4.0 International (CC BY-NC-ND 4.0) License. 